\title{
ON RINGS OF OPERATORS
}

\section{BY P. PORCELLI AND E. A. PEDERSEN}

Communicated by E. Hewitt, July 29, 1966

Let $\mathfrak{H C}$ be a complex Hilbert Space, $B(\mathcal{F C})$ the ring of bounded operators on $\mathfrak{F}, E$ an abelian symmetric subring of $B(\mathfrak{F C})$ containing the identity which is closed in the weak operator topology, $E_{1}$ the commutant of $E$, and suppose $E_{1}$ has a cyclic vector $\xi_{0}$ which we normalize so that $\left|\xi_{0}\right|=1$. Diximier [1] has shown that $E$ (respect. $E_{1}$ ), as a Banach space, is the dual of the Banach space $R$ (respect. $R_{1}$ ) of all linear forms on $E$ (respect. $E_{1}$ ) that are continuous in the ultra-strong topology of $E$ (respect. $E_{1}$ ). In this note we show that every $T \in R$ is also continuous in the weak operator topology of $E$, from which it follows that a linear functional $T$ on $E$ is continuous in either the weak, ultraweak, strong, or ultrastrong topologies if and only if it is continuous in all four simultaneously. In the process, we obtain an integral representation for such $T$, which we later use in a theorem on centrally reducible positive functionals on $E_{1}$.

We denote the maximal ideal space of $E$ by $M$, and for $A, B, \ldots$ $\in E$, we denote the corresponding Gel'fand transforms by $a, b, \cdots$. Then $A \rightarrow a$ is an isometric isomorphism from $E$ onto $C(M)$. Consequently, every bounded linear functional on $E$ has the form

$$
T(A)=\int_{M} a(m) d \nu(m),
$$

where $\nu$ is a complex Borel measure on $M$ uniquely determined by $T$. Of special interest are functionals of the form $A \rightarrow(A \xi, \xi)$, where $\xi$ is a vector of $\mathfrak{H}$. We denote by $\nu_{\xi}$ the measure corresponding to the vector $\xi$, and by $\mu$ the measure $\nu_{\xi 0}$. Then the $\nu_{\xi}$ are all nonnegative, $\left\|v_{\xi}\right\|=|\xi|^{2}$, and it can be seen that $\nu_{\xi} \ll \mu$ for every $\xi$. The space $M$ is extremely disconnected, and the measure $\mu$ has the special property that $C(M) \cong L_{\infty}(M, \mu)$ under the natural embedding.

Theorem 1. A linear functional $T$ on $E$ is ultrastrongly continuous if, and only if it is weakly continuous, and if and only if there exists a $\phi \in L_{1}(M, \mu)$ such that

$$
T(A)=\int_{M} a(m) \phi(m) d \mu(m)
$$

for every $A \in E$.

1 This research was supported by the Air Force Office of Scientific Research, Grant AF-49(638)-1426. 
Indication of Proof. If $T$ is ultrastrongly continuous, then $T$ is norm continuous, hence we have the representation (1). Using the definitions of the ultrastrong topology and the above mentioned fact that $\nu_{\xi} \ll \mu$ for all $\xi$, it follows that $\nu \ll \mu$, hence we have the representation (2).

To prove that a $T$ of the form (2) is weakly continuous, we may consider only the case where $\phi \geqq 0$. For $n=1,2, \cdots$, we define $b_{n}(m)=\phi(m)$ if $n-1 \leqq \phi(m)<n, b_{n}(m)=0$ otherwise. Then $b_{n} \in L_{\infty}(M, \mu)$ for each $n$. Using the fact that $C(M) \cong L_{\infty}(M, \mu)$, we may redefine each $b_{n}$ on a null set $[\mu]$, and obtain the properties $b_{n} \in C(M), b_{n} \cdot b_{m}=0$ if $\dot{n} \neq m, b_{n} \geqq 0, \sum b_{n}=\phi$ a.e. [ $\mu$ ]. For each $n$, let $B_{n} \in E$ with Gel'fand transform $b_{n}$. Then $B_{n} \geqq 0$, and it follows readily

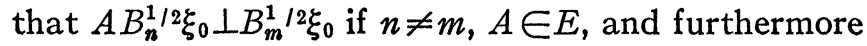

$$
\begin{aligned}
\sum\left|B_{n}^{1 / 2} \xi_{0}\right|^{2} & =\sum\left(B_{n} \xi_{0}, \xi_{0}\right) \\
& =\sum \int_{M} b_{n}(m) d \mu(m) \\
& =\int \phi(m) d \mu(m)<\infty .
\end{aligned}
$$

Setting $\eta=\sum_{n} B_{n}^{1 / 2} \xi_{0}$, we have for $A \in E$,

$$
\begin{aligned}
T(A) & =\int a(m) \phi(m) d \mu(m) \\
& =\sum_{n} \int a(m) b_{n}(m) d \mu(m) \\
& =\sum_{n}\left(A B_{n} \xi_{0}, \xi_{0}\right) \\
& =(A \eta, \eta),
\end{aligned}
$$

hence $T$ is weakly continuous. Since weak continuity trivially implies ultrastrong continuity, the theorem follows.

Recall that a positive functional $T_{1}$ on $E_{1}$ is called centrally reducible if its restriction $T$ to $E$ has the following property: if $T^{\prime}$ is a positive functional on $E$ such that $T^{\prime} \leqq T$, then there exists a $B \in E$ such that $T^{\prime}(A)=T(A B)$ for every $A \in E$.

THEOREM 2. If $T_{1}$ is a weakly continuous positive functional on $E_{1}$, then $T_{1}$ is centrally reducible.

Proof. The restriction $T$ of $T_{1}$ to $E$ is also weakly continuous, hence it has the form (2), where $\phi \geqq 0$. If $0 \leqq T^{\prime} \leqq T$, let $\nu^{\prime}$ be the 
measure corresponding to $T^{\prime}$ in the representation (1). It follows that $0 \leqq \nu(E) \leqq \int_{M} X_{E}(m) \phi(m) d \mu(m)$, hence there exists $b \in L_{\infty}(M, \mu)$ such that $d \nu^{\prime}=b \phi d \mu$. We may assume $b$ continuous, and letting $B$ be the operator of $E$ having Gel'fand transform $b, T^{\prime}(A)=T(A B)$ for arbitrary $A \in E$.

\section{REFERENCE}

1. J. Dixmier, Les algèbres d'opérateurs dans l'espace Hilbertien (Algèbres de von Neumann), Gauthier-Villars, Paris, 1957.

Louisiana State University 